PROCEEDINGS OF THE

AMERICAN MATHEMATICAL SOCIETY

Volume 125, Number 11, November 1997, Pages 3355-3362

S 0002-9939(97)04152-X

\title{
A MONOTONEITY PROPERTY OF THE GAMMA FUNCTION
}

\author{
G. D. ANDERSON AND S.-L. QIU
}

(Communicated by Hal L. Smith)

\begin{abstract}
In this paper we obtain a monotoneity property for the gamma function that yields sharp asymptotic estimates for $\Gamma(x)$ as $x$ tends to $\infty$, thus proving a conjecture about $\Gamma(x)$.
\end{abstract}

\section{INTRODUCTION}

For real and positive values of $x$ the Euler gamma function $\Gamma$ and its logarithmic derivative $\Psi$, the so-called digamma function, are defined as

$$
\Gamma(x)=\int_{0}^{\infty} t^{x-1} e^{-t} d t, \psi(x)=\frac{\Gamma^{\prime}(x)}{\Gamma(x)} .
$$

For extensions of these functions to complex variables and for basic properties see [WW].

Over the past half century many authors have obtained inequalities for these important functions (see [A1], [A2] and bibliographies in those papers). In keeping with this tradition we here obtain a monotoneity property of the gamma function that yields a sharp asymptotic estimate for $\Gamma(x)$ as $x$ tends to $\infty$.

In [AVV, Lemma 2.39] the following result was obtained.

\subsection{Lemma.}

$$
\lim _{x \rightarrow \infty} \frac{\log \Gamma\left(1+\frac{x}{2}\right)}{x \log x}=\frac{1}{2},
$$

and

$$
f(x) \equiv \frac{1}{x} \log \Gamma\left(1+\frac{x}{2}\right)
$$

is strictly increasing from $[2, \infty]$ onto $[0, \infty)$.

It was conjectured in $[\mathrm{AVV}$, Remark 2.41] that the function in (1.3) is strictly increasing from $[2, \infty)$ onto $[0,1 / 2)$. In order to obtain an affirmative answer to the above conjecture we prove here the following result.

1.5. Theorem. The function $f(x) \equiv(\log \Gamma(x+1)) /(x \log x)$ is strictly increasing from $(1, \infty)$ onto $(1-\gamma, 1)$, where $\gamma$ is the Euler-Mascheroni constant. In particular, for $x \in(1, \infty)$,

$$
x^{(1-\gamma) x-1}<\Gamma(x)<x^{x-1}
$$

Received by the editors June 20, 1996.

1991 Mathematics Subject Classification. Primary 33B15; Secondary 26A48, 26D07.

Key words and phrases. Gamma function, psi function, monotoneity, inequalities.

(C)1997 American Mathematical Society 
and

$$
\lim _{x \rightarrow \infty} \frac{\log \Gamma(x)}{(x-1) \log (x-1)}=1 .
$$

In $[\mathrm{AVV}]$ the the following result was also obtained.

1.8. Lemma. Let $\Omega_{n}=\pi^{n / 2} / \Gamma(1+n / 2)$ denote the $n$-dimensional volume of the unit ball $B^{n}$ in $\mathbb{R}^{n}$. Then

$$
\begin{gathered}
\lim _{n \rightarrow \infty} \Omega_{n}^{1 /(n \log n)}=e^{-\frac{1}{2}} \\
\Omega_{n}^{1 / n} \quad \text { decreases strictly to } 0 \quad \text { as } n \rightarrow \infty, \\
\sum_{n=2}^{\infty} \Omega_{n}^{1 / \log n} \text { is convergent. }
\end{gathered}
$$

It was pointed out in [AVV, Remark 2.41] that if the function in (1.3) above has the conjectured property this would imply that $\Omega_{n}^{1 /(n \log n)}$ is strictly decreasing for $n \geq 2$. Thus our Theorem 1.5 implies this monotoneity of $\Omega_{n}^{1 /(n \log n)}$ (see Corollary 3.1). It should be observed that $\Omega_{n}$ itself is not monotone [BH, pp. 263, 264] (cf. $[\mathrm{SV}])$.

In this paper we let $\mathbb{N}$ denote the set of positive integers and, for the real number $x$, let $[x]$ denote the integer satisfying $x-1<[x] \leq x$.

\section{Preliminary Results}

Before establishing the main theorem we need to prove some technical lemmas.

2.1. Lemma. The function $f(x) \equiv \sum_{n=1}^{\infty} \frac{n-x}{(n+x)^{3}}$ is positive for $x \in[1,4)$.

Proof. Let $u(t, x)=(t-x) /(t+x)^{3}$, for $t, x \in[1, \infty)$. Then $u$ is strictly decreasing in $t$ on $[2 x, \infty)$ for any $x \in[1, \infty)$, since $\partial u / \partial t=2(2 x-t) /(x+t)^{4}$.

For $k \in \mathbb{N}$ and $x \geq 1$, let $v_{k}=u(k, x)$. Then $v_{k}(x)$ is strictly decreasing in $k$ for $k \in \mathbb{N} \cap[2 x, \infty)$ and

$$
v_{k}(x)=u(k, x)>u(t, x) \text { for } t>k \geq 2 x .
$$

Hence, for $k \geq 2 x \geq 2$,

$$
v_{k}(x)=\frac{k-x}{(k+x)^{3}}=\int_{k}^{k+1} v_{k}(x) d t>\int_{k}^{k+1} u(t, x) d t,
$$

so that

$$
\left\{\begin{array}{r}
\sum_{n=2[x]+2}^{\infty} v_{n}(x)>\sum_{n=2[x]+2}^{\infty} \int_{n}^{n+1} u(t, x) d t=\int_{2[x]+x}^{\infty} \frac{t-x}{(t+x)^{3}} d t \\
=\int_{2[x]+2}^{\infty}\left[\frac{1}{(t+x)^{2}}-\frac{2 x}{(t+x)^{3}}\right] d t=\frac{2(1+[x])}{(2[x]+x+2)^{2}}
\end{array}\right.
$$


It follows from (2.2) that

$$
\begin{aligned}
f(x) & =\sum_{n=1}^{2[x]+1} v_{n}(x)+\sum_{n=2[x]+2}^{\infty} v_{n}(x)>\sum_{n=1}^{2[x]+1} v_{n}(x)+\frac{2(1+[x])}{(2[x]+x+2)^{2}} \\
& =\left[\frac{1-x}{(1+x)^{3}}+\frac{2-x}{(2+x)^{3}}+\cdots+\frac{[x]-x}{([x]+x)^{3}}\right] \\
& +\left[\frac{[x]+1-x}{([x]+x+1)^{3}}+\frac{[x]+2-x}{([x]+x+2)^{3}}+\cdots+\frac{2[x]+1-x}{(2[x]+x+1)^{3}}\right] \\
& \quad+\frac{2(1+[x])}{(2[x]+x+2)^{2}} \\
& \geq \frac{1}{(x+1)^{3}}[(1+x)+(2-x)+\cdots+([x]-x)] \\
+ & \frac{1}{(2[x]+x+1)^{3}}[([x]+1-x)+([x]+2-x)+\cdots+(2[x]+1-x)] \\
& \quad+2 \frac{[x]+1}{(2[x]+x+2)^{2}} \\
& \equiv \frac{[x]([x]+1-2 x)}{2(x+1)^{3}}+\frac{([x]+1)(3[x]+2-2 x)}{2(2[x]+x+1)^{3}}+\frac{2[x]+1}{(2[x]+x+2)^{2}} \\
f_{1}(x) . &
\end{aligned}
$$

If $1 \leq x<2$, then $[x]=1$ and

$$
\left\{\begin{aligned}
f_{1}(x) & =\frac{1-x}{(x+1)^{3}}+\frac{5-2 x}{(3+x)^{3}}+\frac{4}{(4+x)^{2}} \\
& =\frac{1}{(x+1)^{3}(4+x)^{2}}\left[3 x^{3}+5 x^{2}+4 x+20\right]+\frac{5-2 x}{(3+x)^{3}}>0 .
\end{aligned}\right.
$$

If $2 \leq x<3$, then $[x]=2$ and

$$
\left\{\begin{aligned}
f_{1}(x) & =\frac{3-2 x}{(x+1)^{3}}+3 \frac{4-x}{(5+x)^{3}}+\frac{6}{(6+x)^{2}} \\
& =\frac{4 x^{3}-3 x^{2}-18 x+114}{(x+1)^{3}(6+x)^{2}}+3 \frac{4-x}{(5+x)^{3}} \\
& >\frac{4 x^{3}+33}{(x+1)^{3}(6+x)^{2}}+3 \frac{4-x}{(5+x)^{3}}>0 .
\end{aligned}\right.
$$


If $3 \leq x<4$, then $[x]=3$ and

$$
\left\{\begin{aligned}
f_{1}(x) & =\frac{3(2-x)}{(x+1)^{3}}+\frac{2(11-2 x)}{(7+x)^{3}}+\frac{8}{(8+x)^{2}} \\
& =\frac{5 x^{3}-18 x^{2}-72 x+392}{(x+1)^{3}(8+x)^{2}}+2 \frac{11-2 x}{(7+x)^{3}} \\
& >\frac{1}{(8+x)^{2}}\left[\frac{5 x^{3}-18 x^{2}-72 x+392}{(x+1)^{3}}+\frac{22-4 x}{7+x}\right] \\
& =\frac{x^{4}+27 x^{3}-144 x^{2}-50 x+2766}{(x+8)^{2}(x+7)(x+1)^{3}} \\
& >\frac{1072}{(x+8)^{2}(x+7)(x+1)^{3}}>0 .
\end{aligned}\right.
$$

The conclusion now follows from $(2.2)-(2.5)$.

2.6. Lemma. The function $g(x) \equiv x^{2} \Psi^{\prime}(1+x)-x \Psi(1+x)+\log \Gamma(1+x)$ is positive for all $x \in[1, \infty)$.

Proof. From the well-known difference equation $\Gamma(x+1)=x \Gamma(x)[\mathrm{WW}$, p. 237] it follows easily that

$$
\Psi(x+1)=\frac{1}{x}+\Psi(x)
$$

from which we obtain

$$
x g^{\prime}(x)=x^{2} \Psi^{\prime}(x)+x^{3} \Psi^{\prime \prime}(x)+1 \equiv g_{1}(x) .
$$

Since

$$
\Psi^{\prime}(x)=\sum_{n=0}^{\infty} \frac{1}{(x+n)^{2}}, \Psi^{\prime \prime}(x)=-2 \sum_{n=0}^{\infty} \frac{1}{(x+n)^{3}}
$$

[Ah, p. 200, (31)], $g_{1}$ can be rewritten as

$$
g_{1}(x)=x^{2} \sum_{n=1}^{\infty} \frac{n-x}{(n+x)^{3}}=x^{2} f(x),
$$

where $f$ is as in Lemma 2.1.

From Lemma 2.1, (2.8), and (2.9), we see that $g$ is strictly increasing on $[1,4]$, and hence, by [W, Exercise 2, p. 80] and [Ah, p. 199, (29)],

$$
g(x) \geq g(1)=\Psi^{\prime}(2)-\Psi(2)=\sum_{n=2}^{\infty} \frac{1}{n^{2}}-1+\gamma=\frac{\pi^{2}}{6}+\gamma-2=0.2221 \cdots>0
$$

for $x \in[1,4]$.

Next, since

$$
\left\{\begin{array}{l}
\frac{1}{x}<\Psi^{\prime}(x)<\frac{1}{x-1} \\
\log x-\frac{1}{x}<\Psi(x)<\log x-\frac{1}{2 x}
\end{array}\right.
$$


for $x>1$ (see [ABRVV, Theorem 3.1], [S, Lemma 4b], [A2, (2.2)]), it follows from (2.7) that

$$
\left\{\begin{array}{r}
g(x)=x^{2} \Psi^{\prime}(x)-x \Psi(x)+\log x+\log \Gamma(x)-2 \\
>x+(1-x) \log x+\log \Gamma(x)-\frac{3}{2} \equiv g_{2}(x) .
\end{array}\right.
$$

Differentiation gives

$$
g_{2}^{\prime}(x)=\Psi(x)-\left(\log x-\frac{1}{x}\right), x>1,
$$

which is positive by $(2.11)$. Hence $g_{2}$ is strictly increasing on $[1, \infty)$ so that, for $x \in[4, \infty)$,

$$
g(x)>g_{2}(x) \geq g_{2}(4)=\frac{5}{2}-3 \log 4+\log 6=0.1328 \cdots>0 .
$$

The result now follows from (2.10) and (2.13).

2.14. Lemma. The function $h(x) \equiv x \Psi(1+x)-\log \Gamma(1+x)$ is strictly increasing from $[0, \infty)$ onto $[0, \infty)$. Moreover,

$$
\lim _{x \rightarrow \infty} \frac{h(x)}{x}=1 \quad \text { and } \quad \frac{h(x)}{x}=1+\mathrm{O}\left(\frac{\log x}{x}\right)
$$

as $x \rightarrow \infty$.

Proof. Differentiation gives

$$
h^{\prime}(x)=x \Psi^{\prime}(x+1)>0,
$$

and the monotoneity of $h$ follows. Clearly, $h(0)=0$. Since

$$
\left\{\begin{array}{l}
\log \Gamma(x)=\left(x-\frac{1}{2}\right) \log x-x+\frac{1}{2} \log (2 \pi)+\mathrm{O}\left(\frac{1}{x}\right), \\
\Psi(x)=\log x-\frac{1}{2 x}+\mathrm{O}\left(\frac{1}{x^{2}}\right)
\end{array}\right.
$$

as $x \rightarrow \infty$ by [S, Theorems 4,5$]$, we have

$$
\begin{aligned}
\lim _{x \rightarrow \infty} \frac{h(x)}{x} & =\lim _{x \rightarrow \infty}\left[\log (1+x)-\frac{1}{2(x+1)}-\frac{1}{x}\left(x+\frac{1}{2}\right) \log (x+1)+\frac{x+1}{x}\right] \\
& =\lim _{x \rightarrow \infty}\left[1-\frac{\log (1+x)}{2 x}\right]=1 .
\end{aligned}
$$

2.17. Lemma. The function $H(x) \equiv \log x-\frac{1}{h(x)} \log \Gamma(x+1)$ is strictly increasing from $[1, \infty)$ onto $[0,1)$. Here $h$ is as in Lemma 2.14. In particular, for all $x \in$ $(1, \infty)$,

$$
1-\frac{1}{\log x}<\frac{\log \Gamma(x+1)}{x \Psi(x+1)}<1-\frac{1}{1+\log x}
$$


Proof. Clearly, $H(1)=0$. It follows from (2.15) and (2.16) that

$$
\begin{aligned}
\lim _{x \rightarrow \infty} H(x) & =\lim _{x \rightarrow \infty}\left[\left(1-\frac{x}{h(x)}\right) \log x+\frac{x}{h(x)}\left(-\frac{\log x}{2 x}+1-\frac{\log (2 \pi)}{2 x}+\mathrm{O}\left(\frac{1}{x^{2}}\right)\right)\right] \\
& =\lim _{x \rightarrow \infty}\left[\mathrm{O}\left(\frac{(\log x)^{2}}{h(x)}\right)+\frac{x}{h(x)}+\mathrm{O}\left(\frac{1}{x}\right)\right] \\
& =1+\lim _{x \rightarrow \infty} \mathrm{O}\left(\frac{1}{x}(\log x)^{2}\right)=1 .
\end{aligned}
$$

Next, by differentiation, we get

$$
\begin{aligned}
H^{\prime}(x)= & \frac{1}{x}-\frac{1}{(h(x))^{2}}\left[\Psi(x+1) h(x)-x \Psi^{\prime}(x+1) \log \Gamma(x+1)\right] \\
= & \frac{1}{x}-\frac{1}{x(h(x))^{2}}[(x \Psi(x+1)-\log \Gamma(x+1)+\log \Gamma(x+1)) h(x) \\
& \left.\quad-x^{2} \Psi^{\prime}(x+1) \log \Gamma(x+1)\right] \\
= & \frac{\log \Gamma(x+1)}{x(h(x))^{2}}\left[x^{2} \Psi^{\prime}(x+1)-h(x)\right] \\
= & \frac{g(x)}{x(h(x))^{2}} \log \Gamma(x+1),
\end{aligned}
$$

where $g$ is as in Lemma 2.6. Hence the monotoneity of $H$ follows from Lemma 2.6. The inequality (2.18) is clear.

\section{Proof of the Main Theorem}

We now show how Theorem 1.5 follows from the lemmas in Section 2. By differentiation we get

$$
(x \log x)^{2} f^{\prime}(x)=h(x) H(x),
$$

where $f(x)=(\log \Gamma(x+1)) /(x \log x)$ and where $h$ and $H$ are as in Lemmas 2.14 and 2.17 , respectively. Hence, the monotoneity of $f$ follows from Lemmas 2.14 and 2.17 .

Next, by l'Hôpital's Rule, we have

$$
f\left(1^{+}\right)=\lim _{x \rightarrow 1} \frac{\Psi(x+1)}{1+\log x}=\Psi(2)=-1-\gamma
$$

[AS, 6.3.3] and, by (2.16),

$$
\begin{aligned}
\lim _{x \rightarrow \infty} f(x) & =\lim _{x \rightarrow \infty} \frac{\left(x+\frac{1}{2}\right) \log (x+1)-(x+1)+\frac{1}{2} \log (2 \pi)}{x \log x} \\
& =\lim _{x \rightarrow \infty} \frac{\log (x+1)}{\log x}=1 .
\end{aligned}
$$

Inequality (1.6) and limit (1.7) are clear. 
3.1. Corollary. (1) The function $f(x) \equiv\left(\log \Gamma\left(1+\frac{x}{2}\right)\right) /(x \log x)$ is strictly increasing from $[2, \infty)$ onto $[0,1 / 2)$ (the conjecture in $[\mathrm{AVV}$, Remark 2.41] is true).

(2) For $n \in \mathbb{N}$, let $\Omega_{n}=\pi^{n / 2} / \Gamma(1+n / 2)$ be the $n$-dimensional volume of the unit ball $B^{n}$ in $\mathbb{R}^{n}$. Then the sequence $G(n) \equiv \Omega_{n}^{1 /(n \log n)}$ is strictly decreasing for $n \geq 2$, with $G(2)=\pi^{1 / \log 4}$ and $\lim _{n \rightarrow \infty} G(n)=e^{-1 / 2}$.

Proof. (1) Let $t=x / 2$. Then

$$
f(x)=\frac{\log t}{2 \log (2 t)} \cdot \frac{\log \Gamma(t+1)}{t \log t},
$$

and the conclusion follows from Theorem 1.5 since the function $(\log t) / \log (2 t)$ is strictly increasing from $[1, \infty)$ onto $[0,1)$.

(2) Since

$$
\log G(n)=\frac{1}{2} \frac{\log \pi}{\log n}-\frac{\log \Gamma\left(1+\frac{n}{2}\right)}{n \log n},
$$

the assertion follows from part (1).

3.2. Remark. By methods similar to those used to prove Theorem 1.5 we can show that the function $f(x) \equiv(\log \Gamma(x+1)) /((x-1) \log (2 x))$ is strictly increasing from $[4.5, \infty)$ onto $[c, 1)$, where $c=(\log (135 \sqrt{\pi} / 32)) /(7 \log 3)$. However, $f$ is not monotone on $(1, \infty)$ since $f^{\prime}(x)<0$ when $x$ is near 1 .

3.3. Conjecture. $f(x) \equiv(\log \Gamma(x+1)) /(x \log x)$ is concave on $(1, \infty)$.

\section{ACKNOWLEDGEMENTS}

This paper was completed during the second author's visit to Michigan State University. The authors thank Dr. M. Vuorinen for helpful comments.

\section{REFERENCES}

[AS] M. Abramowitz and I. A. Stegun, editors, Handbook of Mathematical Functions with Formulas, Graphs and Mathematical Tables, Dover, New York, 1965. MR 31:1400

[Ah] L. V. Ahlfors, Complex Analysis, An introduction to the theory of analytic functions of one complex variable, 3rd ed., McGraw-Hill, New York, 1979. MR 80c:30001

[A1] H. Alzer, Some gamma function inequalities, Math. Comp. 60 (1993), 337-346. MR 93f:33001

[A2] H. Alzer, On some inequalities for the gamma and psi functions, Math. Comp. 66 (1997), 373-389. MR 97e:33004

[ABRVV] G. D. Anderson, R. W. Barnard, K. C. Richards, M. K. Vamanamurthy, and M. Vuorinen, Inequalities for zero-balanced hypergeometric functions, Trans. Amer. Math. Soc. 347 (1995), 1713-1723. MR 95m:33002

[AVV] G. D. Anderson, M. K. Vamanamurthy, and M. Vuorinen, Special functions of quasiconformal theory, Exposition. Math. 7 (1989), 97-136. MR 90k:30032

[BH] J. Böhm and E. Hertel, Polyedergeometrie $n$-dimensionalen Räumen Konstanter Krümmung, Birkhäuser, Basel - Boston - Stuttgart, 1981. MR 82k:52001a

[S] J. Sandor, Sur la fonction gamma, Publ. Centre Rech. Math. Pures (I) (Neuchâtel) 21 (1989), 4-7.

[SV] D. J. Smith and M. K. Vamanamurthy, How small is a unit ball?, Math. Mag. 62 (1989), 101-107. MR 90e:51029 
[W] H. F. Weinberger, Partial Differential Equations, John Wiley \& Sons, New York, 1965.

[WW] E. T. Whittaker and G. N. Watson, A Course of Modern Analysis, Cambridge Univ. Press, Cambridge, 1958. MR 31:2375

Department of Mathematics, Michigan State University, East Lansing, Michigan 48824

E-mail address: anderson@math.msu.edu

School of Science and Arts, Hangzhou Institute of Electronics Engineering (HiEe), Hangzhou 310037, People's Republic of China 